\title{
Analytical calculation of the combined suspension truss
}

\author{
Mikhail Kirsanov ${ }^{1, *}$, Dmitriy Tinkov ${ }^{1}$ and Oleh Boiko ${ }^{1}$ \\ ${ }^{1}$ National Research University "Moscow Power Engineering Institute", Krasnokazarmennaya 14, \\ Moscow, 111250, Russia
}

\begin{abstract}
An algorithm is given for obtaining the formula for the dependence of the deflection of a regular planar truss of an arched type with a suspended lower belt on the number of panels. The cases of uniform loading of the nodes of the upper and lower belts by a vertical load are considered. To generalize a number of solutions obtained in the system of computer mathematics Maple to an arbitrary case, an induction method was applied. For this purpose, for a sequence of coefficients of the particular solutions found, a common term is determined which is a solution of the recurrence equation. The deflection was determined with the help of Mohr's integral, which depends on the forces in the rods. Forces in a statically determinate construction were performed by cutting out nodes from the solution of a system of equations written in a matrix form. The analytical dependence of displacement of the mobile support on the number of panels is found.
\end{abstract}

\section{Introduction}

With the advent in the mid 80s of the last century computer mathematics systems in engineering practice for the calculation of building structures, it became possible to use not only numerical methods [1-9], but also analytical methods. The very first samples in this direction were reduced to obtaining formulas for calculations of deflection and stresses in a concrete design with a parametric representation of the load, dimensions, and elastic properties of the material. The narrow application of such formulas did not satisfy the engineers. Significantly useful formulas are for a wide class of structures, for example, for trusses in which the number of panels is arbitrary. Among other things, such formulas make it possible to find a solution for trusses with a very large number of rods, without a loss of accuracy characteristic of numerical methods. In [10-14], the method of induction of generalization of a series of particular solutions for trusses with different (consecutive) number of panels was used to calculate flat trusses. Operators of the Maple system were used to find symbolic expressions for the stresses in the rods and to compile recurrence equations for the coefficients of the required formulas with their subsequent solution. The method of induction is applicable for regular structures having a periodic structure. The search for schemes of statically determinate trusses was carried out by R. G. Hutchinson

\footnotetext{
* Corresponding author: c216@ya.ru
} 
and N. A. Fleck [15]. In [16-19], the induction method solved more complex problems for spatial constructions, in [19] for the problem of a pile foundation with a regular arrangement of piles. In [20], the method of double induction (based on the number of panels in height and the number of panels along the length of the span) was used to calculate the planar truss. A brief review of the analytical solutions of problems on the deflection of flat trusses is given in [21].

\section{Truss and Methods}

The basis of the construction is an arched truss with a height of $n h$ and a span (distance between supports) $2 n h$. The lower belt is suspended on vertical hangers from $h$ to $n h$.

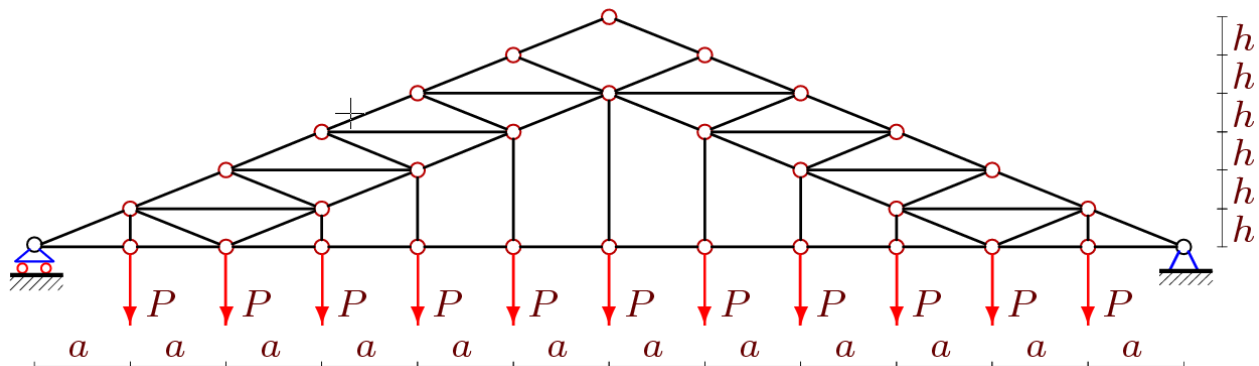

Fig. 1. Truss, load on the lower belt $n=6$

To calculate the deflection of the truss and obtain an analytical form of the solution, it is necessary to obtain expressions for the forces in the rods as well in symbolic form. To do this, using the program [10-13], you need to enter data about the coordinates of the nodes of the truss and the order of connecting the rods. We number nodes and rods, choosing the origin of coordinates in the left movable hinged support (Fig. 2).

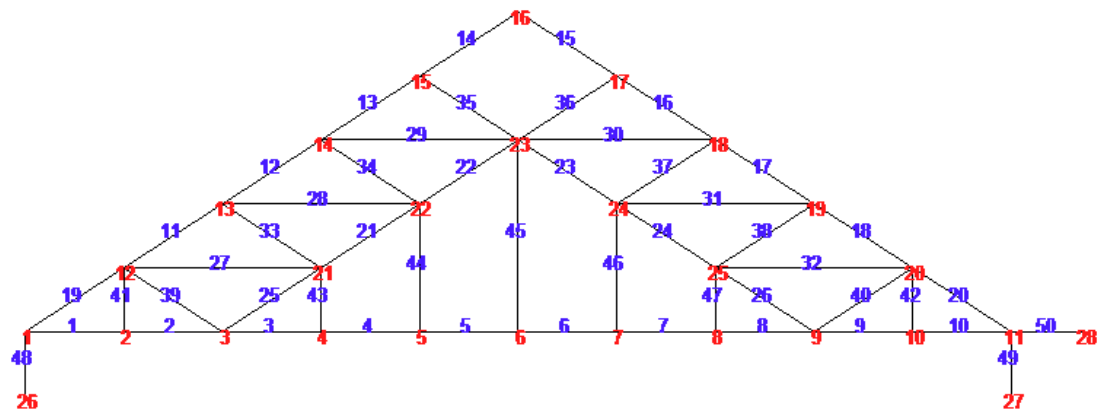

Fig. 2. Numbering rods and hinges in the Maple system, $n=5$

In the language of symbolic mathematics Maple this has the form

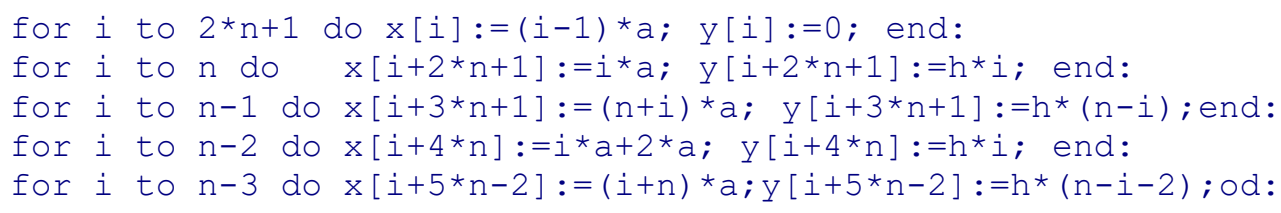


The order of connecting the rods are recorded in special lists containing the numbers of their ends. For rods of the lower and upper belts, this looks like this:

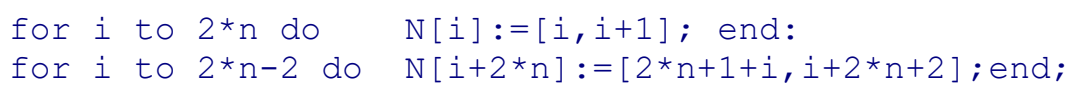

The matrix $G$ of the system of equilibrium equations $G \bar{S}=\bar{B}$ for nodes consisting of equations in the projections on the axis is filled with the direction cosines of the forces. In odd rows, projections are recorded on the horizontal axis, in even lines - on the vertical [13], $\bar{S}-$ is the vector of all forces in the rods. The displacement is obtained from the Mohr's integral

$$
\Delta=\sum_{i=1}^{m-3} S_{i}^{(P)} S_{i}^{(1)} l_{i} /(E F) .
$$

It is indicated: $S_{i}^{(1)}$ — the forces from the unit force applied to the knot of the lower belt in the middle of the span, $E F$ - the rigidity of the rods, $S_{i}^{(P)}$ - the forces in the rods from the given load, $l_{i}$ - the length of the rods.

\section{Results}

Based on the results of calculations of several trusses, we obtain that the form of the solution is the following

$$
\Delta=P\left(A a^{3}+C c^{3}+H h^{3}\right) /\left(2 h^{2}(n-2)\right) .
$$

where $c=\sqrt{a^{2}+h^{2}}, n>2$. A rare feature of this solution is the presence of the number of panels $n$ in the denominator of the formula. All known solutions [10-13,15-20] have the form of polynomials of different degrees. The calculation of a series of trusses reveals sequences of coefficients for $a^{3}, h^{3}, c^{3}$. Operators of the Maple system rgf_findrecur and rsolve from the solution of recurrence equations give the following patterns

$$
\begin{aligned}
& A=n^{3}+4 n^{2}+6 n-12, \\
& C=\left(4 n^{3}-13 n^{2}+33 n-36\right) / 6, \\
& H=2(n-2)^{2}, n=3,4,5, \ldots .
\end{aligned}
$$

Using the operator rgf_findrecur(4,[ 69, 140, 243, 384, 569, 804, 1095, 1448], A, n), the arguments of which are the terms of the sequence of coefficients at $a^{3}$ the recurrence equation of the fourth order is received

$$
A_{n}=4 A_{n-1}-6 A_{n-2}+4 A_{n-3}-A_{n-4} .
$$

The solution of this equation is given by the operator rsolve.

For the coefficient $C$ we have the same equation $C_{n}=4 C_{n-1}-6 C_{n-2}+4 C_{n-3}-C_{n-4}$.

For the coefficient $H$, equation

$$
H_{n}=3 H_{n-1}-3 H_{n-2}+H_{n-3}
$$

is obtained. We note that the coefficients of the recurrence equations are binomial, but in general for other induction problems this assertion is valid. 
Expression (2,3) gives the solution of the problem posed for the load along the upper belt of the truss.

Similarly, for the load on the upper belt of the truss (Figure 3), we obtain the deflection in the form (1) and the coefficients

$$
\begin{aligned}
& A=n^{3}+4 n^{2}-4 n-4, \\
& C=\left(2 n^{3}-4 n^{2}-n+6\right) / 3, \\
& H=0 .
\end{aligned}
$$

The load on the upper belt of the truss does not involve vertical suspensions, with a length proportional to $h$, that remain unloaded. Hence it turns out that the coefficient $H$ for $h^{3}$ vanishes.

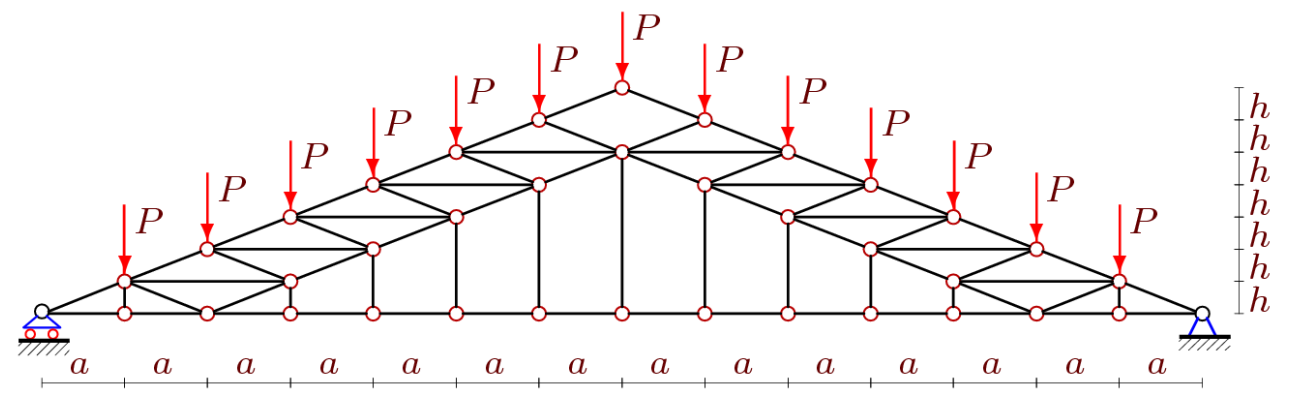

Fig. 3. Truss, load on the upper belt $n=7$

The simpler coefficients in (1) will be in calculating the deflection from one force in the middle of the lower belt (Figure 4):

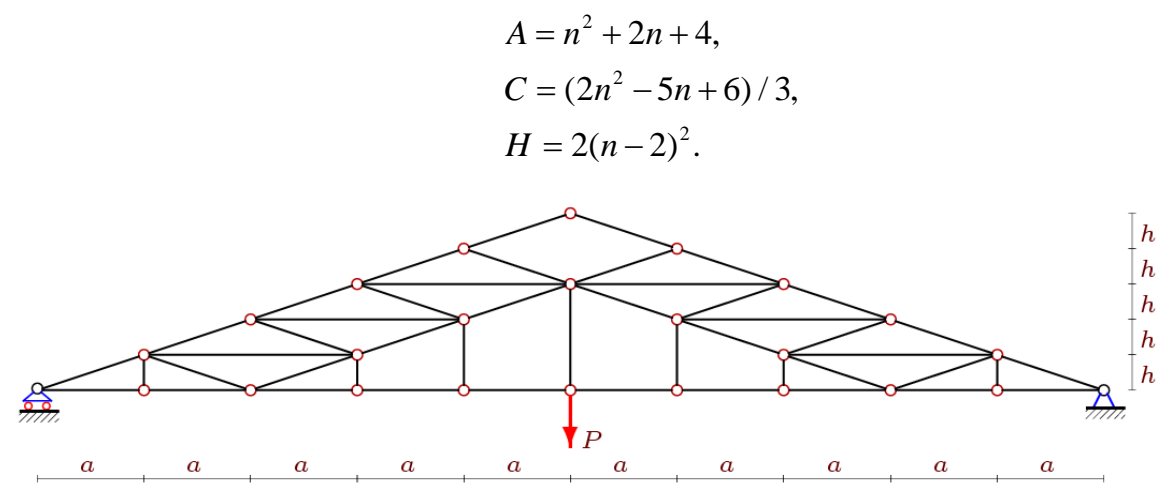

Fig. 4. Truss, load on the center, $n=5$

\section{Discussion}

The analytical form of the solution makes it possible to easily estimate the deflection of the structure, to select the optimal number of panels and the dimensions of the structure. We construct a graph of the solution of the problem of the deflection of a truss under the action of a load along the lower belt. We introduce the dimensionless deflection $\Delta^{\prime}=\Delta E F /\left(P_{0} L\right)$. Figure 5 shows the curves of the obtained dependence at 
$L=2 n a=60 m, P_{0}=P(2 n-1)$. An oblique asymptote is obvious, which is also easily obtained by means of Maple $\lim _{n \rightarrow \infty} \Delta^{\prime} / n=h /(6 L)$.

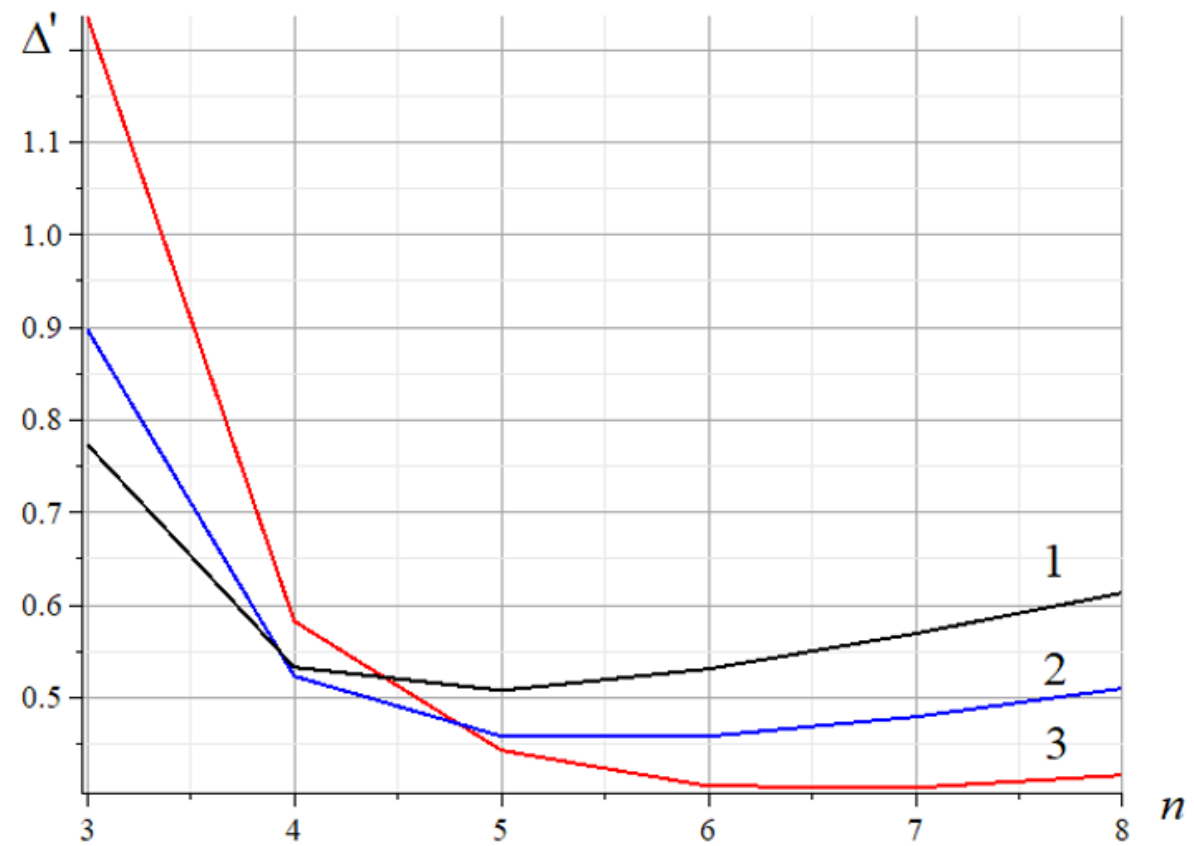

Fig 5. Dependence of the dimensionless deflection on the number of panels during loading on the lower belt. $1-h=20.0 \mathrm{~m}, 2-h=16.0 \mathrm{~m}, 3-h=12.0 \mathrm{~m}$

All curves show a minimum, allowing to optimize the size of the structure. Points of self-intersections of curves are also interesting. It turns out that for different truss heights with the same number of panels, the relative deflection will be the same.

When the truss is bent under load, the left movable support also moves. To derive the formula for the dependence of this quantity on the number of panels, we repeat the inductive analysis using formula (1), where $S_{i}^{(1)}$ — the forces from the unit horizontal force applied to the left movable hinged support. The following sequence of coefficients is obtained: $19,30,43,58,75,94$. This turned out to be quite enough to obtain the general term of the sequence and the solution for the horizontal displacement

$$
\Delta_{h}=P a^{2}\left(n^{2}+4 n-2\right) /(h E F) .
$$

The curves illustrating this dependence (Figure 6) with a span length of $60 \mathrm{~m}$ show that with an increase in the number of panels, the relative dimensionless displacement $\Delta_{h}{ }^{\prime}=\Delta E F /\left(P_{0} L\right)$ drops to zero. The same confirms the limit $\lim _{n \rightarrow \infty} \Delta_{h}{ }^{\prime}=0$. 


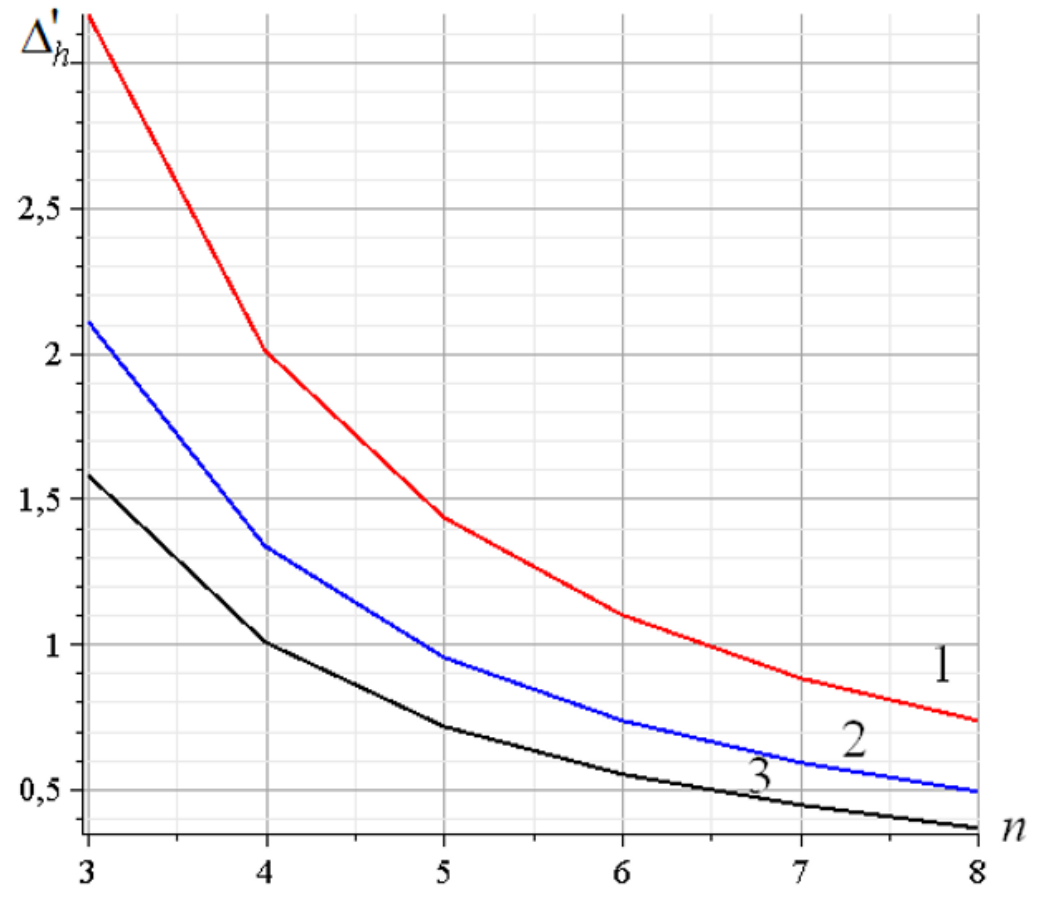

Fig 6. Dependence of the horizontal dimensionless displacement of the support under load on the lower belt ; $L=60 \mathrm{~m} ; 1-h=2 \mathrm{~m} ; 2-h=3 \mathrm{~m} ; 3-h=4 \mathrm{~m}$

When the truss is loaded on the upper belt, the formula for the displacement of the mobile support will differ only by one term:

$$
\Delta_{h}=P a^{2}\left(n^{2}+4 n-4\right) /(h E F) .
$$

And a very simple expression for the displacement of the support will be obtained when solving the problem of the action of one force in the middle of the span (Figure 4):

$$
\Delta_{h}=P a^{2}(n+2) /(h E F) .
$$

\section{Conclusions}

A simple scheme of a plane symmetric combined hanging construction is proposed that allows an analytical solution of the problem of deflection with an arbitrary number of panels. The solution obtained by the induction method has a simple form of a rational function of the number of panels. A remarkable feature of the formulas is that the accuracy of the solution does not depend on the number of panels, while numerical methods after a certain number of rods in the design start to fail, explained by the accumulation of errors. The graphs of the constructed solutions show the presence of extremum points. Some simple asymptotic properties of the solution are obtained. All the formulas derived in the article can be applied in the practice of engineering calculations, and the induction method itself can be applied to virtually any regular structures. A linear combination of solutions obtained under different loads, one can derive formulas for a wide range of problems on deflection and displacement of the support of the truss. 


\section{References}

1. K.A. Egarmin, G.Yu. Sysoev, N.I. Vatin, M.V. Vrublevskaya, Construction of Unique Buildings and Structures 2, 34 (2015)

2. M. Y. Baranovskiy, V. A. Tarasov, Construction of Unique Buildings and Structures 7, 92 (2014)

3. O.A. Sergeyev, V.G. Kiselev and S.A. Sergeyeva, Magazine of Civil Engineering, 1, 74 (2016) doi: 10.5862/MCE.61.7

4. I.N. Serpik, A.V. Alekseytsev, P.Yu. Balabin, N.S. Kurchenk, Magazine of Civil Engineering, 8, 181 (2017) doi: 10.18720/MCE.76.16

5. T. Saknite, D. Serdjuks, V. Goremikins, L. Pakrastins, N.I. Vatin, Magazine of Civil Engineering, 4, 26 (2016) doi: 10.5862/MCE.64.3

6. A. Kaveh, V. R. Mahdavi, Structural Engineering and Mechanics 5, 847 (2015)

7. A. Kaveh, A. Zolghadr, Applied Soft Computing Journal, 5, 2727 (2013)

8. A. Ahrari, D. Kalyanmoy, Computers \& Structures 164, 127 (2016)

9. I.N. Serpik, A.V. Alekseytsev, Magazine of Civil Engineering, 1, 14 (2016) doi: 10.5862/MCE.61.2

10. M.N. Kirsanov, N. Zaborskaya, Magazine of Civil Engineering, 3, 61 (2017) doi: 10.18720/MCE.71.7

11. M.N. Kirsanov, Magazine of Civil Engineering, 1, 33 (2016) doi: 10.5862/MCE.61.4

12. M.N. Kirsanov, Magazine of Civil Engineering, 5, 58 (2015) doi: 10.5862/MCE.57.5

13. M. Kirsanov and D. Tinkov, Analytical calculation of the deflection of the lattice truss, MATEC Web of Conferences, vol. 193, 03015 (2018) doi.org/10.1051/matecconf/201819303015

14. D.V. Tinkov, Magazine of Civil Engineering, 1, 25 (2016) doi: 10.5862/MCE.61.3

15. R. G. Hutchinson, N.A. Fleck, ZAMM Z. Angew. Math. Mech. , 9, 607 (2005).

16. M.N. Kirsanov, Magazine of Civil Engineering, 4, 38 (2015) doi: 10.5862/MCE.56.5

17. M.N. Kirsanov, Magazine of Civil Engineering, 4, 52 (2016) doi: 10.5862/MCE.64.5

18. M.N. Kirsanov, Magazine of Civil Engineering, 8, 58 (2017) doi: 10.18720/MCE.76.6

19. M.N. Kirsanov, Magazine of Civil Engineering, 3, 3 (2015) doi: 10.5862/MCE.55.1

20. V.B. Arutyunyan, Postulat, 7, 5 (2018) http://epostulat.ru/index.php/Postulat/article/view/1740/1774

21. D.V. Tinkov, Magazine of Civil Engineering, 5, 66 (2015) doi: 10.5862/MCE.57.6 